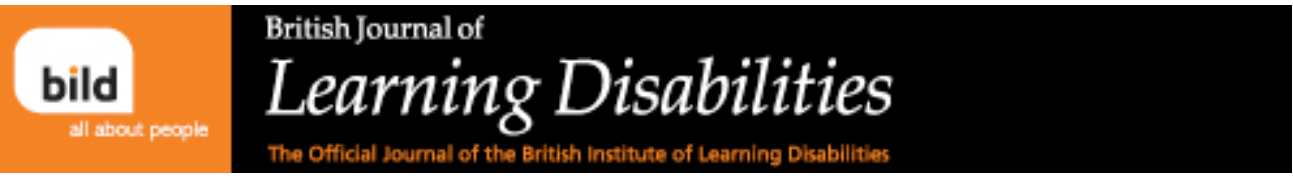

Mental and Physical Health Conditions in People with Intellectual Disabilities: comparing local and national data

\begin{tabular}{|r|l|}
\hline Journal: & British Journal of Learning Disabilities \\
\hline Manuscript ID & BLD-19-0022.R3 \\
\hline Manuscript Type: & Original Article \\
\hline Keywords: & Intellectual disability, Physical Illness, Mental health \\
\hline
\end{tabular}

SCHOLARONE $^{\mathrm{m}}$
Manuscripts $^{\text {Manusion }}$ 


\title{
Mental and Physical Health Conditions in People with Intellectual Disabilities: comparing local and national data
}

Bhathika Perera, Selma Audi, Solomis Solomou, Ken Courtenay, Hugh Ramsay

\author{
Abstract \\ Background:
}

Studies have reported increased prevalence of physical and mental health conditions in people with intellectual disabilities (ID) compared to people without ID. However, there are no studies looking into specific health conditions at a national level and comparing with areas that are socioeconomically disadvantaged (for example low income and low education attainment). This study examines and compares the prevalence of physical and mental health morbidity in people with and without Intellectual disabilities at a local and national level in the UK.

Method:

This study was an anecdotal analysis of physical \& mental health data and Annual Health Checkup for England (national), London (regional) and Haringey (Inner city borough of London) in 2016/17 using data from the NHS Digital database.

Results:

Patterns of mental and physical conditions for people with and without ID was similar across Haringey, London and England data. Severe mental illness was more prevalent in people with ID compared to non ID peers. This further increased in the inner-city London borough for the ID group. Certain physical health conditions were also more prevalent in people with ID. Certain activities such as monitoring blood pressure, recording body mass index and checking $\mathrm{HbA} 1 \mathrm{C}$ were better in people with ID. Uptake of annual health checks for people with ID remained around $50 \%$.

Discussion:

This study further highlights the increased prevalence of mental and physical disorders in people with ID compared to people without ID. Further increased risk of mental disorders in an inner London borough compared to national data aligns with existing literature that highlights the negative impact of socio economic deprivation on mental and physical health. Further studies are 
needed to assess the health and social care measures that can reduce the physical and mental health morbidity in people with ID.

\section{Accessible Summary}

People with intellectual disability have multiple health problems.

People with intellectual disability have more physical and mental health conditions than people without ID.

People with intellectual disability may need more health checks due to increased prevalence of physical and mental illnesses.

More studies are needed to understand what interventions are effective to reduce health problems in people with intellectual disability

\section{INTRODUCTION}

Mental disorders are more prevalent in people with Intellectual Disability (ID) (Cooper, Smiley, Morrison, Williamson, \& Allan, 2007) but rates vary widely between studies. In their systematic review of the prevalence rates of co-occurring psychiatric symptoms or disorders, Buckles et al (2013) reported that the variation in rates was due to the use of different diagnostic criteria and specific samples used in the studies (Buckles, Luckasson, \& Keefe, 2013). Studies at a population level could be considered to give more reliable data on the increased risk of morbidity in the ID population. Analysis of Scottish Census of 2011 data showed that the presence of intellectual disabilities has an odds ratio of 7.1 in predicting mental health conditions adjusted for age and gender (HughesMcCormack et al. 2017). However, some authors have argued that there is no increased risk of mental illness among people with mild ID. Whitaker \& Read (2006) in their systematic review showed that there is no evidence for increased risk of mental illness among people with mild ID (Whitaker \& Read, 2006).

Physical health conditions have a similar increased prevalence rate among people with Intellectual disabilities. Studies over the last four decades have consistently shown that people with intellectual disabilities die prematurely (Glover et al 2017, Forsgren et al, 1996; Forssman \& Åkesson, 2008; Hollins et al 1998). The Confidential Inquiry into Premature Deaths of People with Learning Disabilities (CIPOLD) looked at deaths of people with Intellectual disabilities in South West England (Heslop et al., 


\section{Screening for physical health conditions}

A systematic review of the impact of health checks in multiple countries have shown that they detect serious and unmet health needs in people with ID and potentially improve the knowledge of healthcare professionals on the health needs of people with ID (Robertson, Hatton, Emerson, \& Baines, 2014).

Following recommendations from the Disability Rights Commission (DRC, 2006), the English National Health Service (NHS) introduced an annual health check in Primary Care as a primary intervention to address the health disparity between those with ID and those without ID. A health check was introduced in 2009 along with training for doctors and nurses in GP surgeries.

An analysis of the outcomes of Annual Health Checks by Buszewicz et al, (2014) showed that GP practices incentivised by additional payments for health checks did more health checks leading to further investigations and referrals to secondary care compared to non-incentivised practices (Buszewicz et al., 2014). However, the data does not show if such interventions were effective in reducing morbidity and mortality gap between people with ID and those without ID.

\section{Aims of the study}


A better understanding of the prevalence of physical and mental health disorders in people with Intellectual disabilities and its relationship to Annual Health Checks in England is important to understand current health provision and make recommendations to improve existing care for people with Intellectual disabilities.

Socio-economic \& demographic factors such as poverty, migration and social discrimination are some of the known risk factors for mental and physical health morbidity. The London Borough of Haringey is an extremely diverse borough of London with a high index of deprivation and high unemployment even though the level of deprivation has reduced compared to 2010 data (National Statistics, 2015). Haringey also represents an ethnically diverse community with over a hundred different languages spoken across the borough.

This study aims to look at several indicators related to people with ID in England. First it aims to find the prevalence of mental and physical disorders in people with ID in England and the proportion of people availing of Annual Health Checks. Secondly it looks at whether data for England represents different geographical areas. Therefore this study compared national data for people with and without ID to London and an inner London borough. Haringey was chosen as the inner London borough due its diverse socio-economic and demographic factors that may be particularly relevant to the experiences and social situations of people with intellectual disabilities.

\section{Method}

NHS Digital is the national information and technology partner in the National Health Service in the UK that provides information and data on health and social care in England. Its vision is to harness information and technology to improve care in health and social settings (NHS, 2019). General Practice Extraction Service (GPES) collects data from GP practice IT systems on people with and without ID. This is then reported by NHS Digital. Data used in this study was extracted from NHS digital as provided by GPES. This data was available for each clinical commissioning group (CCG) which are statutory NHS bodies with their own governance arrangements responsible for the planning and commissioning of health care services for people in their local areas. There are 211 CCGs in England, of which 32 are in London. NHS Digital data is available for each CCG.

In this study, the NHS Digital database was used to extract data related to mental health and physical health outcomes in people with and without ID over a twelve-month period as extracted by GPES. Information on patients recorded on each general practice's Learning Disabilities Register that provided data to GPES was included in this analysis. People considered as non LD in this analysis were 
individuals that were not in the GP learning disability register. Data were compared for a 12-month period of 2016/2017.

\section{Measures}

Data on physical and mental health measures and annual health checks for people with ID were examined. Conditions that were looked into are listed in table 1. Data were available for specific physical health conditions and health parameters. Data available to analyse on mental health conditions were broadly on three areas: presence of a severe mental illness, an active diagnosis of depression and dementia.

Data Analysis

Data extracted for NHS digital were analysed using Microsoft Excel. The prevalence of mental and physical health conditions for each CCG was compared between those with and without ID.

\section{Results}

Demographics

The data collected by NHS Digital represents $90 \%(283,556)$ of the overall GP surgery population in Haringey, $79 \%(7,588,027)$ in London, and $57 \%(33,476,783)$ in England.

\section{Prevalence of ID and age distribution}

Of the 283,556 residents in Haringey, $0.38 \%$ had a diagnosis of ID in GP register compared with $0.37 \%$ in London and $0.46 \%$ across England. This shows that the recorded prevalence rates of ID in Haringey and London were lower compared to England data. The proportion of people with ID peaked in 18-24 year age group, with a second smaller peak in 50-54 year old age group (Figure 1 \& 2). The overall age distribution was similar across all England, London, and Haringey.

\section{Uptake of Annual Health Checks}

Annual Health Checks for people with ID are carried out by General Practitioners. Of those recorded with an ID, 54.5\% of people with ID in Haringey received an Annual Health Check compared with London (57.1\%) and England (49.7\%). The data indicates that almost one in two people with ID have had an Annual Health Check with a marginally higher uptake in London and Haringey compared to national figures.

Physical health outcome measures 
Health conditions and health related behaviours such as attending appointments for health screening and having blood pressure checked that were recorded within 15 months to the reference year end date (2017) were included in the data.

Weight

The results are listed in table 1 on physical health measures. A higher proportion (Haringey - 64.1\%, London - 63.1\%, England - 60.3\%) of people with ID had their Body Mass Index (BMI) recorded in GP practices compared to people without ID (Haringey-30.1\%, London 27\%, England- 27.8\%). The percentage with obesity in ID were higher than among people without ID across Haringey, London, and nationally (39.2\% vs $27.7 \%, 36.3 \%$ vs $26.2 \%, 37.9 \%$ vs $30.3 \%)$ Figure 3.

Gastrointestinal conditions

More people with Intellectual disability were reported to have gastro-oesophageal reflux disease (GORD) with rates in Haringey (5.5\%), London (6.9\%) and England (7.8\%) compared with the general population of $0 \%$.

Cardiovascular Diseases

Coronary heart disease (CHD) was higher in people without ID (3.1\%) compared to people with ID (1.1\%). The prevalence rate of hypertension was $10 \%$ for both ID and the non-ID populations in Haringey and London, compared with $13.7 \%$ for the non-ID population in England. The prevalence of heart failure was similar in the ID and the non-ID population in England (0.9\%).

Long-term Health Conditions

The prevalence rates of chronic kidney disease (CKD) in people with ID were $2.9 \%$ (Haringey), $2.3 \%$ (London), and 2.8\% (England). The data indicates that the rates of CKD were higher in the non-ID population in England (3.2\%) compared with Haringey (1.6\%) and London (1.9\%). Interestingly, the prevalence rates of CKD in Haringey and London were higher in the ID population compared to those without ID.

The prevalence rates of Type 1 diabetes in people with ID was 0.5\% (Haringey), 0.6\% (London), and $0.7 \%$ (national) whilst the rates for non-ID population were $0.2 \%$ (Haringey), $0.3 \%$ (London), and $0.4 \%$ (national). Non-type 1 diabetes prevalence rates were similarly higher for people with ID compared to people without ID.

There was a marked difference in the prevalence rates of hypothyroidism; in people with ID the rates were $7.1 \%$ (Haringey), 7.6\% (London) and 8.1\% (England), whereas rates among people without ID were $2.5 \%$ (Haringey), 3.0\% (London) and 3.6\% (England). 
Central nervous system

Recorded epilepsy was the most striking difference between the groups of people with ID compared to people without ID. $17.8 \%$ of people with ID in England had a recorded diagnosis of epilepsy compared to $0.6 \%$ in the non-ID population. In those with ID and epilepsy, $42.2 \%$ were seizure free compared to $58 \%$ of those without ID and with epilepsy. Percentage of people with epilepsy in ID and non ID in Haringey and London were similar to national data.

Screening

The percentage of women with ID eligible for cervical screening ranged from $24 \%$ to $25.1 \%$ for Haringey, London, and England. Out of the eligible group, only $29.6 \%$ to $30.8 \%$ of women had cervical smears done. This significantly contrasts with the non-ID population which had a similar percentage of women eligible for cervical screening ( $25.2 \%$ to $30.2 \%$ ), but $72.8 \%$ to $75.9 \%$ of women had had cervical smear testing.

Mental Health

Table 2 refers to mental illness/disorder in people with and without ID. It indicates that the proportion of people with a diagnosis of severe mental illness was considerably higher compared to those without ID, across Haringey ( $14.1 \%$ vs. $1.3 \%)$, London ( $10.8 \%$ vs. $1.1 \%)$, and nationally ( $8.1 \%$ vs. $0.9 \%)$. Even though the definition of severe mental illness was not defined when extracting data, this data is likely to capture severe mental illnesses such as Schizophrenia and bipolar disorder. People with ID had 9 10.8 times the risk of having a severe mental health diagnosis compared to people without ID. Haringey had the highest risk ratio (10.8) compared to England (9.0).

The prevalence of an active diagnosis of depression in the non-ID population was $12.2 \%$ for England and $8.1 \%$ for Haringey. The prevalence rates of depression in people with ID were $13.2 \%$ for England and $11.1 \%$ for Haringey indicating the risk of developing depression among people with ID is $1.08-$ 1.37 that of people without ID. The prevalence rate for dementia in England for the non-ID population was $0.7 \%$ but was $1.4 \%$ in people with ID. The rates of dementia in both the ID and non-ID populations in London and Haringey were lower than those reported for England. The prevalence rate remained double in people with ID compared to those without ID (risk ratio of 2-2.25).

\section{Discussion}

To our knowledge this is the largest study comparing the physical and mental health data for people with and without ID at a national and local level in England. 
This study found clear differences in the rates of mental and physical disorders between those with ID and those without. This is similar to what was reported in Scotland (Cooper et al 2015). Increased prevalence of physical and mental disorders in people with ID were also reported in Ireland and the Netherlands but in a population of older people with ID (Hermans \& Evenhuis, 2014; McCarron et al., 2013).

Though there were higher rates of certain positive health behaviours such as having Blood Pressure checked and BMI (body mass index) recorded among those with ID, some behaviour such as having cervical screening were low in those with ID.

The higher prevalence rate of mental disorders in people with ID compared to the non-ID population has been repeatedly shown in studies (Cooper \& Bailey, 2001; Hughes-McCormack et al., 2017; Prasher, 1995). This study found a substantially higher prevalence of severe mental illness in people with intellectual disabilities where the rate was higher in London compared to the whole of England. Haringey had a higher rate of severe mental illness compared to London and England. In other words, 128 additional cases of severe mental illness per 1000 people in ID population compared to people without ID in Haringey, versus 72 additional cases for England. There were 60 additional cases of mental health problems in people with ID in Haringey for 1000 population compared to number of cases of severe mental illness in ID for England. This shows the significance of mental health conditions in people with ID compared to non ID population and how it can vary from one area to another. There are many factors that can affect the variable rates in different parts of the country. These may include socio demographic factors, confidence of GPs to make a diagnosis of mental disorder in people with ID and availability of secondary mental health in Intellectual disability services that make mental health diagnoses.

There were clear differences in physical health measures and outcomes in people with ID compared to the non-ID population. Certain physical health conditions were more prevalent in people with ID while there were certain conditions less prevalent in this group compared with non-ID. People with ID are a heterogenous group due to the cause of their ID, therefore it can be argued that certain physical health conditions are associated more with certain forms of ID which can affect the prevalence rate for physical conditions for the whole ID population. For example, Down Syndrome is associated with hypothyroidism and dementia. This study found a similar increased pattern in the prevalence for epilepsy and asthma among people with ID compared to people without ID in keeping with the findings of Cooper et al. (2015) in Scotland. Robertson et al (2014) in their systematic review found a pooled estimate of $22.2 \%$ for epilepsy in people with ID (Robertson et al., 2014). Our study reported a figure of $17.8 \%$ for the data in England. 
Cooper et al. (2015) reported a lower prevalence of dementia in people with ID compared to non-ID population, but the data for England indicates the opposite. Prevalence of dementia appear to be higher in ID population compared to non ID population in this data set.

Prevalence of depression was marginally higher in ID population compared to non ID population for data in England. However, prevalence rate for depression in England was higher than the data for London and Haringey.

Diagnosis of Intellectual Disability (ID) is made using the International Classification of Diseases (World Health Organisation 1992) or Diagnostic Statistical Manual (American Psychiatric Association 2013) criteria. Routine clinical practice suggest that diagnosis of ID is generally made by an assessment of functional abilities along with a developmental history with less emphasis on IQ testing. How the diagnosis of ID made in primary care is not clear. According to previous studies, the prevalence of Intellectual disability is reported to vary from $0.8 \%$ to $3.6 \%$. The reason for the wide variation in prevalence rate is argued to be due to inclusion or exclusion of people with mild ID. The prevalence rate for people with an IQ below 50 that includes moderate to profound ID is $0.25 \%-0.5 \%$. Studies in Scotland have reported a prevalence rate of $0.56 \%$ (Barnett et al., 2012; Cooper et al., 2015). This study showed a prevalence rate of $0.46 \%$ for ID in England which suggests that it reflects people with moderate, severe and profound ID. People with moderate to profound ID are more likely to be recorded in GP practices than people with mild ID given that the diagnosis of ID in primary care is easier when someone has a significantly lower level of intellectual functioning and deficits in adaptive behaviour skills. The diagnosis of mild LD on the other hand can be challenging and may need specific assessments since the abilities of people with mild ID can be overestimated.

The hypothesis that the GP databases may be representing people with IQ below 50 has several implications. Firstly, mental and physical health data for people with ID from this study are more representative of people with more severe ID and less so for people with milder ID. Secondly, it raises the question of whether interventions are needed at the primary care level to identify every person with ID.

The uptake of Annual Health Checks (AHC) remains at approximately $50 \%$. This figure needs to be increased considering the effectiveness of AHC among people with ID in reducing preventable emergency admissions as shown by Carey et al (2017) in their study looking at hospital admissions for people with an intellectual disability from across English GP practices. Authors of this review showed that emergency admissions for conditions that are potentially preventable through better clinical management (e.g.- constipation, inhalation of food) were lower in GP practices that had high uptake 
of annual health checks compared to non-participating practices. There is further increasing emphasis on ambulatory care sensitive conditions through the NHS long term plan 2019.

If this activity is the main strategy to reduce health disparities, further action is needed to understand and overcome barriers to people with ID having their Annual Health Checks done at their GP practice.

\section{Limitations of the study}

This study only includes people who have been diagnosed with ID at primary care. Therefore, there is a risk of missing data on people with ID, especially those with mild ID, not included in the ID data category. The diagnosis of Intellectual disability (Learning disability) in the GP database may also include a broader group of people, including people with specific learning difficulties. Data recorded on GP database may not be accurate as data collected in primary care may lack rigour as occurs in research in which data is collected with a specific aim. GP practices that provided data were not randomly selected in the NHS digital data. Therefore, coverage of data can vary even within one geographical areas. As a result, there needs to be some caution when interpreting data at a local level and less so at a national level.

The absence of data on deprivation and other socio-demographic factors of participants limit the study's ability to understand the reasons behind these differences. A detailed analysis of different areas such as geographically rural parts of the country and comparing the deprivation index is needed to reach any conclusions on the association between mental and physical health problems in people with ID and socio economic \& demographic factors.

Inclusion of data from the GP recording system depends on how GPs record their findings in the electronic data system. If a GP makes an entry using free text rather than following the questionnaire of the Annual Health Check, that information will not be included in the NHS Digital database. GP surgeries also have the facility to change the questions in the Annual Health Check system; ineffective questions could result in some GP surgeries not collecting data, leading to under-reporting parameters. Due to Annual Health Checks, people with ID are more likely to be screened for mental and physical health problems than people without ID. This can affect the results and show higher rates of certain conditions in a screened population than in the non-screened one.

\section{Conclusion}

This is one of the few studies comparing both physical and mental health measures in people with and without intellectual disability for a whole country population, a major metropolitan area and a local, 
well-defined borough in the city. The analysis highlighted on-going disparities in health outcomes between the ID and the non-ID populations. The pattern was similar in England, London, and Haringey but Haringey had significantly higher rates of mental health morbidity in people with ID.

Higher prevalence of various physical and mental health conditions highlights the need for preventative and curative strategies at a population level. Difference in prevalence of mental and physical health conditions in different areas also highlights the importance of assessing local needs when designing services. Effective strategies are needed to increase the uptake of Annual health checks as it is the main method to screen and treat underlying health conditions at present. This study also suggests that most data collected may only reflect people with moderate to profound ID. Studies focusing on people with mild ID are needed, as this group can often fall between services and not receive physical and mental health inputs.

\section{Key Messages}

1. Adults with intellectual disabilities have poor health outcomes resulting in increased morbidity and mortality.

2. Prevalence of mental and physical health conditions vary from one area to another.

3. Further studies are needed to understand the reasons behind different prevalence rates and outcome measures.

4. Effective strategies are needed to increase annual health checks as it is the primary intervention within the NHS to address the health disparity between people with and without intellectual disabilities.

5. People with mild ID are less likely to be captured in these studies, so further studies are needed to understand physical and mental health issues in the mild ID group.

6. Novel approaches in organising primary and secondary care services for people with special requirements needs more emphasis.

Figure 1. Patients registered with Intellectual Disability as a percentage of total practice population 


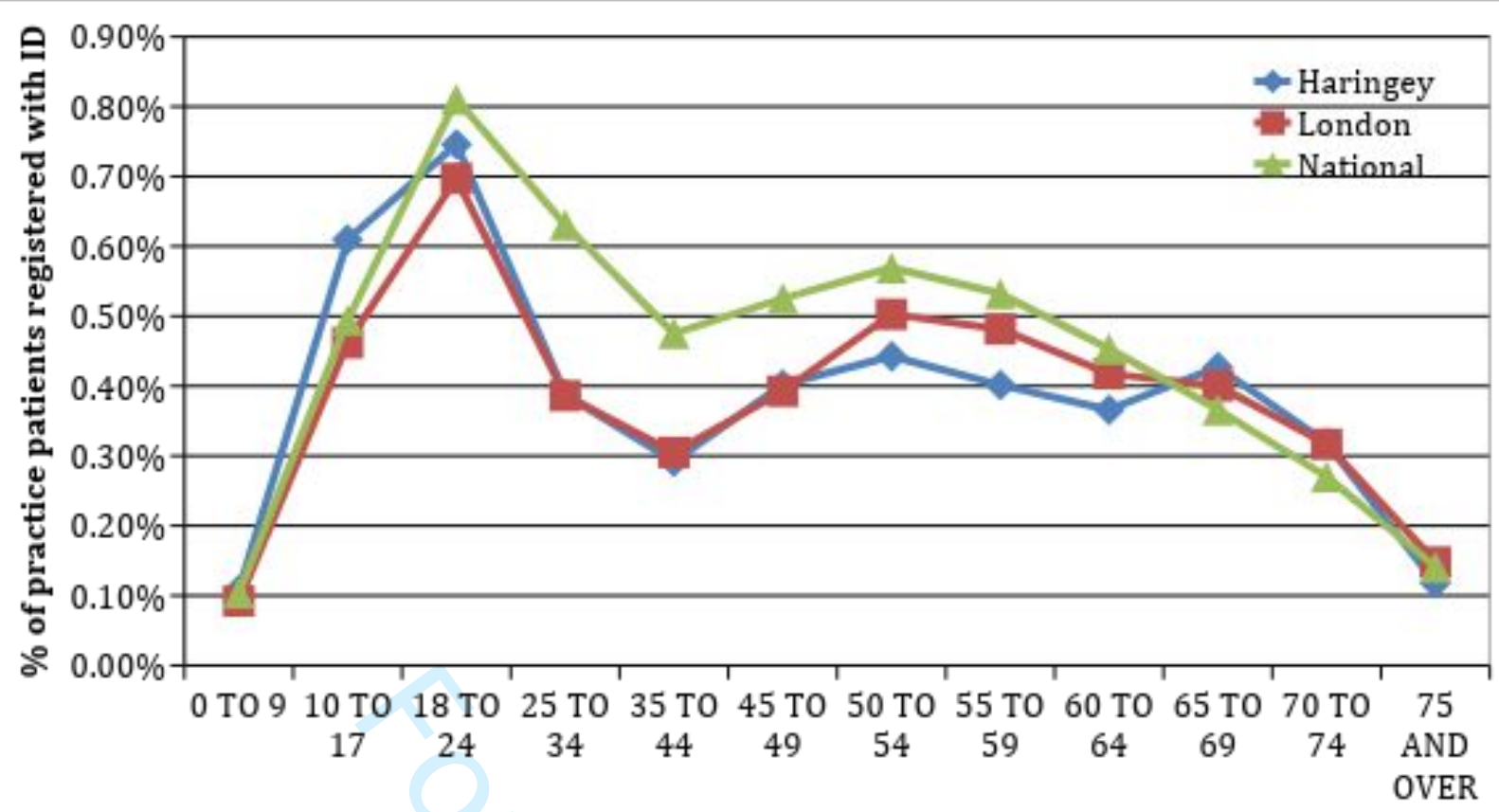

Figure 2- Cumulative distribution of population age group, England, London, Haringey 2016-17

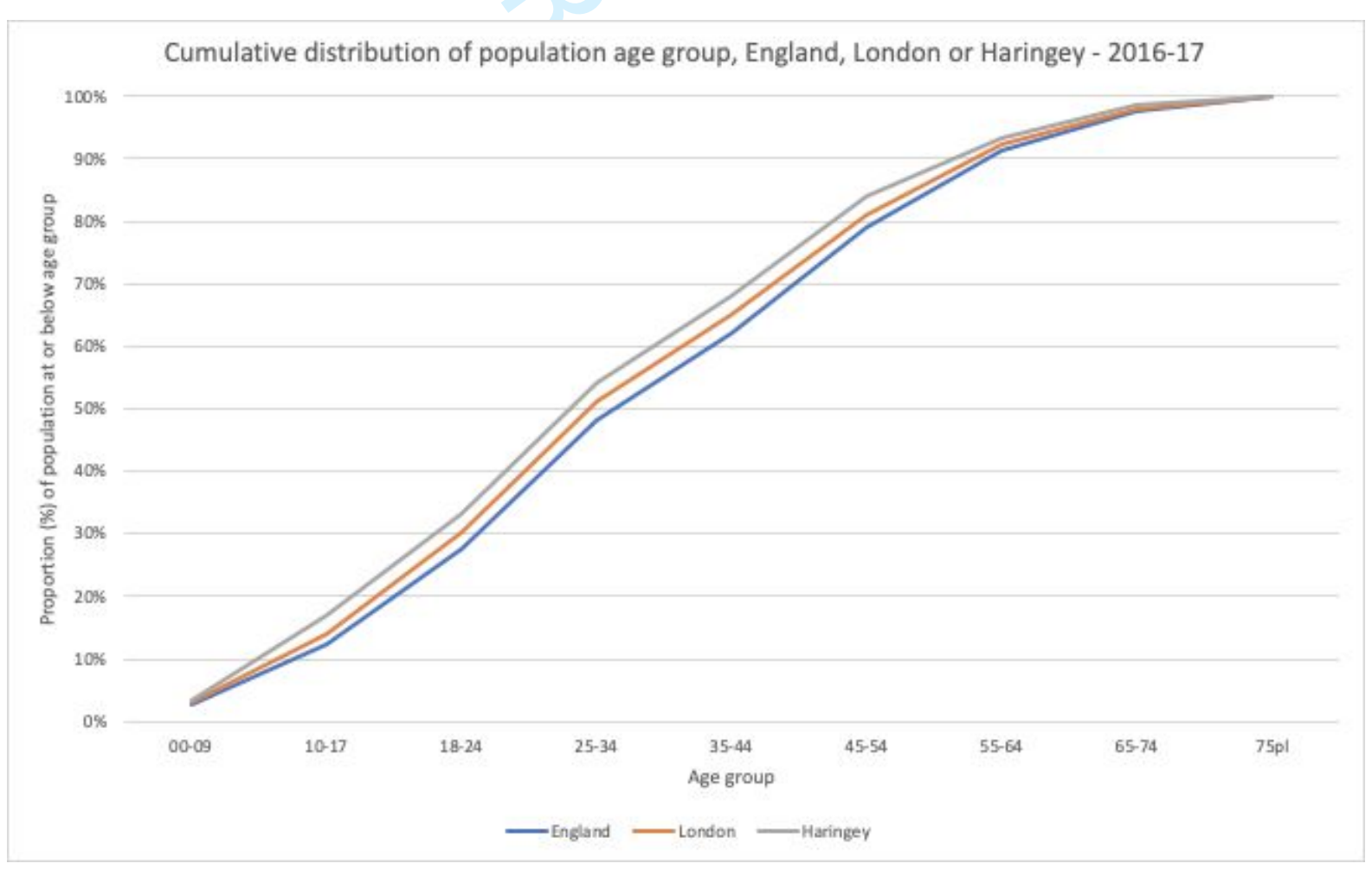

Figure 3. Distribution of BMI in those patients with recorded values 


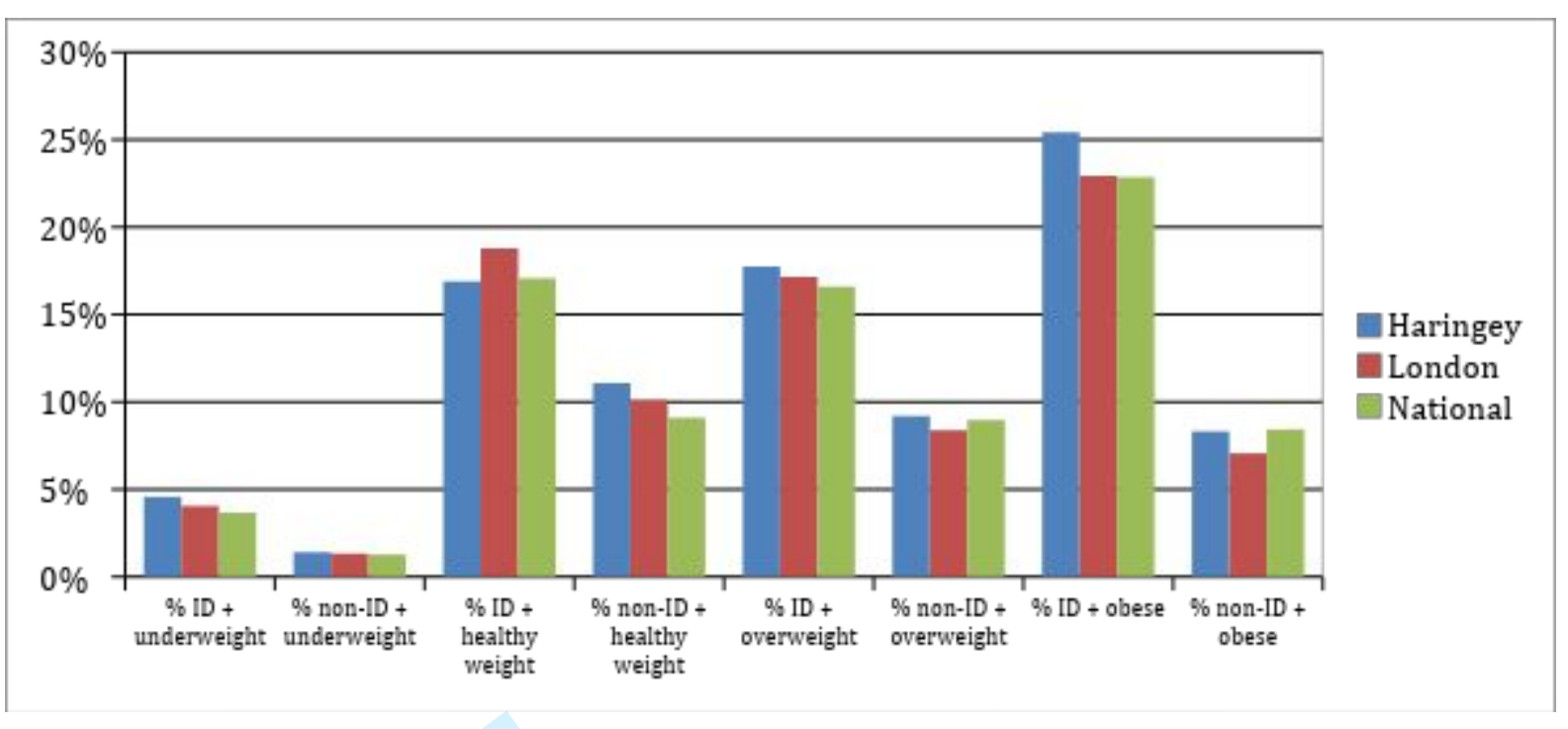

Table 1. Physical health outcome measures

\begin{tabular}{|l|l|l|l|}
\hline & $\begin{array}{l}\text { Haringey } \\
\text { CCG }\end{array}$ & $\begin{array}{l}\text { London } \\
\text { CCGs }\end{array}$ & National \\
\hline Total reported Practice Population & $\mathbf{2 8 3 , 5 5 6}$ & $\mathbf{7 , 5 8 8 , 0 2 7}$ & $\mathbf{3 3 , 4 7 6 , 7 8 3}$ \\
\hline \% of reported population with diagnosis of ID & $0.38 \%$ & $0.37 \%$ & $0.46 \%$ \\
\hline \% ID + Health Check & $54.5 \%$ & $57.1 \%$ & $49.7 \%$ \\
\hline \% ID + BMI recorded & $64.7 \%$ & $63.1 \%$ & $60.3 \%$ \\
\hline \% non-ID + BMI recorded & $30.1 \%$ & $27.0 \%$ & $27.8 \%$ \\
\hline \% ID+ eligible Cervical screen & $24.0 \%$ & $24.6 \%$ & $25.1 \%$ \\
\hline \% non-ID + eligible cervical screen & $30.2 \%$ & $28.3 \%$ & $25.2 \%$ \\
\hline \% ID+ cervical smear & $29.6 \%$ & $30.8 \%$ & $30.4 \%$ \\
\hline \% non-ID + cervical smear & $72.8 \%$ & $72.9 \%$ & $75.4 \%$ \\
\hline \% ID + BP recorded & $85.1 \%$ & $84.1 \%$ & $83.0 \%$ \\
\hline \% non-ID + BP recorded & $66.7 \%$ & $62.4 \%$ & $64.0 \%$ \\
\hline \% ID + GORD & $5.5 \%$ & $6.9 \%$ & $7.8 \%$ \\
\hline \% non-ID + GORD & $0.0 \%$ & $0.0 \%$ & $0.0 \%$ \\
\hline \% ID + Down Syndrome & $9.0 \%$ & $8.3 \%$ & $9.1 \%$ \\
\hline \% ID+ Down Syndrome + Hypothyroid & $20.3 \%$ & $32.4 \%$ & $31.7 \%$ \\
\hline \% ID+ Down Syndrome + Dementia & $0.0 \%$ & $5.1 \%$ & $0.3 \%$ \\
\hline \% ID + Asthma & $7.5 \%$ & $8.2 \%$ & $8.6 \%$ \\
\hline \% non-ID + Asthma & $4.4 \%$ & $4.7 \%$ & $5.9 \%$ \\
\hline \% ID + Cancer & $0.9 \%$ & $1.0 \%$ & $1.1 \%$ \\
\hline \% non-ID + Cancer & $1.4 \%$ & $1.4 \%$ & $2.1 \%$ \\
\hline \% ID + CKD & $2.9 \%$ & $2.3 \%$ & $2.8 \%$ \\
\hline \% non-ID + CKD & $1.6 \%$ & $1.9 \%$ & $3.2 \%$ \\
\hline \% ID + COPD & $1.1 \%$ & $1.0 \%$ & $1.1 \%$ \\
\hline \% non-ID + COPD & $0.9 \%$ & $1.2 \%$ & $1.9 \%$ \\
\hline \% ID + CHD & $0.7 \%$ & $0.9 \%$ & $1.1 \%$ \\
\hline
\end{tabular}




\begin{tabular}{|c|c|c|c|}
\hline$\%$ non-ID + CHD & $1.6 \%$ & $0.0 \%$ & $3.1 \%$ \\
\hline$\%$ ID + T1DM & $0.5 \%$ & $0.6 \%$ & $0.7 \%$ \\
\hline$\%$ non-ID + T1DM & $0.2 \%$ & $0.3 \%$ & $0.4 \%$ \\
\hline$\%$ ID + nonT1 DM & $8.0 \%$ & $7.7 \%$ & $7.0 \%$ \\
\hline \% non-ID + nonT1 DM & $4.9 \%$ & $4.9 \%$ & $5.0 \%$ \\
\hline$\% I D+D M+$ recorded $H b A 1 c$ & $7.2 \%$ & $7.5 \%$ & $7.0 \%$ \\
\hline$\%$ non-ID + DM + recorded HbA1c & $4.6 \%$ & $4.8 \%$ & $5.0 \%$ \\
\hline$\%$ ID + Hba1c satisfactory & $84.4 \%$ & $81.7 \%$ & $82.2 \%$ \\
\hline \% non-ID + Hba1c satisfactory & $83.5 \%$ & $85.4 \%$ & $86.3 \%$ \\
\hline \% ID + Epilepsy & $14.9 \%$ & $16.2 \%$ & $17.8 \%$ \\
\hline \% non-ID + Epilepsy & $0.4 \%$ & $0.4 \%$ & $0.6 \%$ \\
\hline \% ID+epilepsy+recorded seizure frequency & $11.9 \%$ & $21.2 \%$ & $4.0 \%$ \\
\hline$\%$ non-ID + epilepsy +recorded seizure frequency & $7.1 \%$ & $9.0 \%$ & $0.1 \%$ \\
\hline \% ID+epilepsy seizure free & $36.8 \%$ & $34.0 \%$ & $42.2 \%$ \\
\hline \% non-ID+epilepsy seizure free & $51.1 \%$ & $48.3 \%$ & $58.0 \%$ \\
\hline$\%$ ID + heart failure & $0.9 \%$ & $0.8 \%$ & $0.9 \%$ \\
\hline$\%$ non-ID + heart failure & $0.6 \%$ & $0.6 \%$ & $0.9 \%$ \\
\hline$\%$ ID + HTN & $10.6 \%$ & $10.5 \%$ & $9.9 \%$ \\
\hline$\%$ non-ID + HTN & $10.5 \%$ & $11.1 \%$ & $13.7 \%$ \\
\hline$\%$ ID + stroke or TIA & $1.1 \%$ & $1.5 \%$ & $1.7 \%$ \\
\hline$\%$ non-ID + stroke or TIA & $0.9 \%$ & $1.1 \%$ & $1.7 \%$ \\
\hline$\%$ ID + hypothyroid & $7.1 \%$ & $7.6 \%$ & $8.1 \%$ \\
\hline \% non-ID + hypothyroid & $2.5 \%$ & $3.0 \%$ & $3.6 \%$ \\
\hline
\end{tabular}

Table 2. Mental health outcome measures

\begin{tabular}{|l|l|l|l|}
\hline & $\begin{array}{l}\text { Haringey } \\
\text { CCG }\end{array}$ & $\begin{array}{l}\text { London } \\
\text { CCGs }\end{array}$ & National \\
\hline \% ID + Mental Health & $14.1 \%$ & $10.8 \%$ & $8.1 \%$ \\
\hline \% non-ID + Mental Health & $1.3 \%$ & $1.1 \%$ & $0.9 \%$ \\
\hline \% ID + Dementia & $0.9 \%$ & $1.2 \%$ & $1.4 \%$ \\
\hline \% non-ID + Dementia & $0.4 \%$ & $0.5 \%$ & $0.7 \%$ \\
\hline \% ID + Depression & $11.1 \%$ & $11.4 \%$ & $13.2 \%$ \\
\hline \% non-ID + Depression & $8.1 \%$ & $8.3 \%$ & $12.2 \%$ \\
\hline
\end{tabular}

Bibliography

Ali, A., \& Hassiotis, A. (2008). Illness in people with intellectual disabilities. BMJ (Clinical Research Ed.), 336(7644), 570-571. https://doi.org/10.1136/bmj.39490.543137.80 
American Psychiatric Association. (2013). Diagnostic and statistical manual of mental disorders, 5th ed. Washington, DC: American Psychiatric Association.

Barnett, K., Mercer, S. W., Norbury, M., Watt, G., Wyke, S., \& Guthrie, B. (2012). Epidemiology of multimorbidity and implications for health care, research, and medical education: a crosssectional study. The Lancet, 380(9836), 37-43. https://doi.org/10.1016/S0140-6736(12)60240-2

Buckles, J., Luckasson, R., \& Keefe, E. (2013). A systematic review of the prevalence of psychiatric disorders in adults with intellectual disability, 2003-2010. Journal of Mental Health Research in Intellectual Disabilities, 6(3), 181-207. https://doi.org/10.1080/19315864.2011.651682

Buszewicz, M., Welch, C., Horsfall, L., Nazareth, I., Osborn, D., Hassiotis, A., ... Strydom, A. (2014). Assessment of an incentivised scheme to provide annual health checks in primary care for adults with intellectual disability: a longitudinal cohort study. The Lancet. Psychiatry, 1(7), 522530. https://doi.org/10.1016/\$2215-0366(14)00079-0

Carey, I. M., Hosking, F. J., Harris, T., DeWilde, S., Beighton, C., Shah, S. M., \& Cook, D. G. (2017). Do health checks for adults with intellectual disability reduce emergency hospital admissions? Evaluation of a natural experiment. Journal of Epidemiology and Community Health, 71(1), 5258. https://doi.org/10.1136/jech-2016-207557

Cooper, S.-A., \& Bailey, N. M. (2001). Psychiatric disorders amongst adults with learning disabilities prevalence and relationship to ability level. Irish Journal of Psychological Medicine, 18(2), 4553. https://doi.org/10.1017/S0790966700006315

Cooper, S.-A., McLean, G., Guthrie, B., McConnachie, A., Mercer, S., Sullivan, F., \& Morrison, J. (2015). Multiple physical and mental health comorbidity in adults with intellectual disabilities: population-based cross-sectional analysis. BMC Family Practice, 16, 110. https://doi.org/10.1186/s12875-015-0329-3

Cooper, S.-A., Smiley, E., Morrison, J., Williamson, A., \& Allan, L. (2007). Mental ill-health in adults with intellectual disabilities: prevalence and associated factors. The British Journal of Psychiatry, 190, 27-35. https://doi.org/10.1192/bjp.bp.106.022483 
DRC. (2006). Equal Treatment: Closing the Gap. Disability Rights Commission.

Forsgren, L., Edvinsson, S. O., Nyström, L., \& Blomquist, H. K. (1996). Influence of epilepsy on mortality in mental retardation: an epidemiologic study. Epilepsia, 37(10), 956-963.

Forssman, H., \& Åkesson, H. O. (2008). Mortality of the mentally deficient: a study of 12,903 institutionalised subjects. Journal of Intellectual Disability Research, 14(4), 276-294. https://doi.org/10.1111/j.1365-2788.1970.tb01127.x

Glover, G., Williams, R., Heslop, P., Oyinlola, J., and Grey, J. ( 2017) Mortality in people with intellectual disabilities in England. Journal of Intellectual Disability Research, 61: 62- 74

Hermans, H., \& Evenhuis, H. M. (2014). Multimorbidity in older adults with intellectual disabilities. Research in Developmental Disabilities, 35(4), 776-783. https://doi.org/10.1016/j.ridd.2014.01.022

Heslop, P., Blair, P. S., Fleming, P., Hoghton, M., Marriott, A., \& Russ, L. (2014). The Confidential Inquiry into premature deaths of people with intellectual disabilities in the UK: a populationbased study. The Lancet, 383(9920), 889-895. https://doi.org/10.1016/S0140-6736(13)62026-7

Hollins, S., Attard, M. T., von Fraunhofer, N., McGuigan, S., \& Sedgwick, P. (1998). Mortality in people with learning disability: risks, causes, and death certification findings in London. Developmental Medicine and Child Neurology, 40(1), 50-56.

Hughes-McCormack, L. A., Rydzewska, E., Henderson, A., MacIntyre, C., Rintoul, J., \& Cooper, S.-A. (2017). Prevalence of mental health conditions and relationship with general health in a wholecountry population of people with intellectual disabilities compared with the general population. BJPsych Open, 3(5), 243-248. https://doi.org/10.1192/bjpo.bp.117.005462

McCarron, M., Swinburne, J., Burke, E., McGlinchey, E., Carroll, R., \& McCallion, P. (2013). Patterns of multimorbidity in an older population of persons with an intellectual disability: results from the intellectual disability supplement to the Irish longitudinal study on aging (IDS-TILDA). Research in Developmental Disabilities, 34(1), 521-527. https://doi.org/10.1016/j.ridd.2012.07.029 National Statistics. (2015). English indices of deprivation 2015 - GOV.UK. Retrieved March 24, 2019, 
from https://www.gov.uk/government/statistics/english-indices-of-deprivation-2015 NHS. (2019). NHS Digital. Retrieved March 9, 2019, from https://digital.nhs.uk/ NHS, The NHS Long Term Plan 2019 London Prasher, V. P. (1995). Prevalence of psychiatric disorders in adults with Down syndrome., 9, 77-82. Robertson, J., Hatton, C., Emerson, E., \& Baines, S. (2014). The impact of health checks for people with intellectual disabilities: an updated systematic review of evidence. Research in Developmental Disabilities, 35(10), 2450-2462. https://doi.org/10.1016/j.ridd.2014.06.007 Royal College of General Practitioners. (2019). Health checks for people with learning disabilities toolkit. Retrieved March 9, 2019, from https://www.rcgp.org.uk/clinical-andresearch/resources/toolkits/health-check-toolkit.aspx

Whitaker, S., \& Read, S. (2006). The Prevalence of Psychiatric Disorders among People with Intellectual Disabilities: An Analysis of the Literature. Journal of Applied Research in Intellectual Disabilities, 19(4), 330-345. https://doi.org/10.1111/j.1468-3148.2006.00293.x

World Health Organization. (1992). The ICD-10 classification of mental and behavioural disorders: Clinical descriptions and diagnostic guidelines. Geneva: World Health Organization. 